tion and tracking of trans-national transmission events. We have activated a decentralized multinational network of surveillance nodes. This network simultaneously analyze the cross-border distribution of relevant strains by means of sharing the same set of strain-specific PCRs. Once new cases infected by the surveyed strains are captured by the strain-specific PCRs the isolates are characterized by WGS. From these data we to define in detail the network of relationships between the involved cases. It allows us to differentiate transmissions after arrival of migrants to the host countries from independent importations from their countries of origin. Integrative multinational efforts supported on novel simplified strategies can transform the way in which we survey TB transmission in a new global scenario.

Funding: ERANET-LAC and ISCIII (TRANS-TB-TRANS REF AC16/00057; ELAC2015/T08-0664), FIS (15/01554; 18/00599).

6.18 doi: 10.15789/2220-7619-2018-4-6.18

\section{GENETIC DIVERSITY AND DRUG RESISTANCE OF MYCOBACTERIUM AVIUM IN ITALY}

F. Genua, M. Menichini, N. Lari, L. Rindi

Department of Translational Research, University of Pisa, Pisa, Italy

Mycobacterium avium complex is responsible for most of the human-associated nontuberculous mycobacteria infections. M. avium is classified into 4 subspecies, each endowed with specific pathogenetic and host range characteristics; among these, M. avium subsp. hominissuis (MAH), that is usually isolated from human and swine sources, is an important pathogen that causes infections in the respiratory tract, lymph node, and, occasionally, soft tissue of immunocompetent patients; moreover, it causes disseminated diseases in patients with human immunodeficiency virus infection. In Italy, as in many other countries worldwide, MAH is the most common cause of nontuberculous mycobacteria infection and the incidence of MAH infections is increasing. In the present study, we determined the VNTR-based genetic diversity of a collection of $71 \mathrm{MAH}$ human strains isolated from 2010 to 2016 in order to estimate the genetic relationships among MAH isolates in our setting. Moreover, we performed the clarithromycin susceptibility test in order to investigate whether there was any association between the VNTR pattern and the minimal inhibitory concentration (MIC) of clarithromycin. The VNTR analysis revealed 24 distinct VNTR patterns; of these, 16 patterns were unique, while 8 patterns were shared by 2 or more isolates, thus yielding 8 lusters including a total of 55 isolates. Our results showed that most MAH isolates displayed a close genetic relationship, indicating that the MAH genotypes are quite homogeneous in our geographical area. Such genotypic stability of the MAH strain population circulating in our region supports the hypothesis of the presence of possible local sources of infection and transmission pathways at the local level.

Clarithromycin showed strong antimicrobial activity against MAH isolates, as indicated by the high proportion $(94.4 \%)$ of susceptible strains. No significant association between VNTR genotype and MIC of clarithromycin was found; moreover, due to the small number of resistant isolates, it was not easy to evaluate the correlation between VNTR genotypes and clarithromycin susceptibility.

Further investigations on larger collections of MAH strains of human, animal and environmental origin, are needed both to define the correlation between geno- types and clinical features or drug resistance and to clarify the sources of infection and the specific transmission pathways of our region, in order to achieve a better control of MAH infection.

GENOTYPES OF MYCOBACTERIUM TUBERCULOSIS ISOLATES FROM DIFFERENT ORGANS OF PATIENTS WITH GENERALIZED TB AND HIV-COINFECTION

A.A. Gerasimova ${ }^{1}$, A.A. Vyazovaya ${ }^{1}$, M.Yu. Mayskaya ${ }^{2}$, I.V. Mokrousov' ${ }^{1}$ O.V. Narvskaya ${ }^{1,3}$

${ }^{1}$ St. Petersburg Pasteur Institute, St. Petersburg, Russia $;{ }^{2}$ City Pathoanatomical Bureau, St. Petersburg, Russia; ${ }^{3}$ Research Institute of Phthisiopulmonology, St. Petersburg, Russia

The purpose of the study was to genotype Mycobacterium tuberculosis isolates from internal organs of patients from St. Petersburg, Russia, with generalized tuberculosis (TB) and HIV coinfection, to assess possible association between the M. tuberculosis genotype and localization of TB disease.

A total of 128 strains of $M$. tuberculosis were recovered from 55 patients with HIV infection of stages 4 or 5 and generalized TB with multiple lesions of internal organs (from 2 to 10), were studied. Most of the patients had affected lungs (50 cases), intrathoracic and intra-abdominal lymph nodes (46 and 34 cases respectively), spleen (40 cases), kidneys (32 cases), brain and meninges (32 cases). M. tuberculosis isolates were cultured from lungs (48), intrathoracic and intra-abdominal lymph nodes (40), spleen (20), kidneys (14), meninges and brain (7). M. tuberculosis were isolated from one affected organ in 11 patients, and in the remaining cases isolates from 2 or 3 affected organs (16 and 28 persons, respectively) were obtained. Genotyping was performed by spoligotyping (all strains) and IS6110-RFLP typing (Beijing genotype), the obtained profiles were compared with the international database SITVIT_WEB and a proprietary database (Narvskaya, 2003), respectively. The data were subjected to statistical analysis.

$67.3 \%$ (37 of 55) patients were infected by M. tuberculosis Beijing genotype. Of the 37 patients infected with Beijing strains, strains of cluster A0 were isolated from 14 patients, B 0 from 5 patients, and 10 other RFLP types were obtained in the remaining 18 patients. The non-Beijing genotypes were represented by LAM (4), Ural (5), T (5) and 4 others (1 strain of each genotype), all of which belong to the Euro-American lineage of M. tuberculosis (lineage 4). No difference was observed for isolates from different organs of the same patient. Of the 37 patients infected with Beijing strains, the lungs were affected in 33 patients, intrathoracic lymph nodes in 28 , spleen in 28 , intra-abdominal lymph nodes in 28 , kidneys in 24 , and brain and meninges in 24 patients. Of 18 patients infected with non-Beijing strains, lungs were affected in 17 patients, intrathoracic lymph nodes in 18 , spleen in 12 , intraabdominal lymph nodes in 11 , kidneys in 8 , brain and meninges in 10. Comparison of different organs for association with infection by Beijing and non-Beijing strains did not reveal statistically significant differences: lungs $(P=0.890)$; intrathoracic lymph nodes $(\mathrm{P}=0.503)$; spleen $(\mathrm{P}=0.777)$; intra-abdominal lymph nodes $(\mathrm{P}=0.970)$; kidneys $(\mathrm{P}=$ $0.448)$; brain and meninges $(\mathrm{P}=0.886)$.

Beijing genotype was predominant among $M$. tuberculosis isolates studied. Among Beijing family strains, cluster A0 (corresponding to the Central Asian Russian strain) prevailed. There were no statistically significant differences between Beijing and any of non-Beijing genotypes with regard to frequency of isolation from particular organs. 